\title{
THREE-DIMENSIONAL ANALYSIS OF SANDWICH STRUCTURES BY BOUND- ARY ELEMENT METHOD
}

\author{
F. P. A. Almeida ${ }^{1}$, R. S. Romão Filho ${ }^{2}$ \\ ${ }^{1}$ Laboratory of Scientific Computing and Visualization, Federal University of Alagoas (pat- \\ rick@lccv.ufal.br)
}

${ }^{2}$ São Carlos School of Engineering, University of São Paulo

\begin{abstract}
The development of new materials has become increasingly important in the growth of various industrial sectors, awakening the interest of the aeronautical, naval, automobile, offshore, sports and civil engineering industries. These materials include composites, which if well designed, enable the best characteristics of their constituent parts to be exploited. However, along with all of its benefits, such materials pose significant challenges when it comes to determining their mechanical properties. Thanks to advances in technology and the constant evolution of computers, these properties can be determined numerically through such methods as the Finite Element Method (FEM) and the Boundary Element Method (BEM), among others. This study seeks to determine the linear mechanical behaviour of heterogeneous structures through computer simulations, using a three-dimensional BEM/BEM coupling formulation, where the sub-region technique is applied. Linear approximation for triangular boundary elements is also considered. The effective mechanical properties of the sandwich structures obtained by the Boundary Element Method and the Mixing Rule were compared, as well as the relationships between stiffness and weight in the sandwich structures and in the homogeneous plates. In all of the analyzed cases, the percentage error between the methods was found to be high, with some reaching over 50\%. This can be attributed to the simplicity of the Mixing Rule, where the Poisson ratio of the constituent materials is not taken into consideration. In other words, the strains resulting from the Poisson effect are constant throughout the material. With respect to the study of the relationship between stiffness and weight in sandwich structures and in comparison with equivalent homogeneous structures, it was found that in all of the cases, the thickness of the homogeneous material was lower than that of the composite material. Materials with metal foam (light aluminum) display a higher stiffness-to-weight ratio than the homogeneous plates. Those containing polyurethane have the lowest stiffness-to-weight ratio.
\end{abstract}

Keywords: Boundary Element Method, Sandwich structures, Three-dimensional analysis. 


\section{INTRODUÇÃO}

O rápido avanço tecnológico tem motivado uma crescente necessidade do emprego de materiais com avançado desempenho e características desejáveis em importantes áreas da ciência e nos mais diversos setores industriais. Este fato tem proporcionado um desenvolvimento substancial na tecnologia de fabricação e projeto de novos materiais e nas investigações teóricas e experimentais de seus comportamentos. Dentro de uma importante classe destes modernos materiais encontram-se aqueles que apresentam uma microestrutura heterogênea, constituída pela combinação estratégica de diferentes fases e da qual resulta um material composto com desempenho superior ao de seus componentes. Dentre estes materiais destacam-se os compósitos reforçados por fibras que, devido as suas interessantes características, encontram a cada dia novas aplicações tecnológicas. Para justificar a importância atual e futura de tais materiais heterogêneos, pode-se mencionar o grande interesse por eles despertado nas indústrias aeronáutica, naval, automobilística, esportiva, offshore e da construção civil.

A palavra compósito significa dois ou mais materiais que combinados em uma escala macroscópica formam um terceiro material único com fases distintas e desempenho diferente daquele exibido pelos seus constituintes individualmente. Uma grande vantagem dos compósitos é que quando bem projetados, exibem melhores qualidades que seus constituintes, assim como características desejáveis não apresentadas pelos materiais individualmente. Dentro desta definição, enquadram-se, por exemplo, os materiais reforçados por fibras e os materiais particulados, como concretos e argamassas, os quais são constituídos por uma matriz envolvendo fibras ou inclusões (partículas) que, normalmente, servem como materiais de reforço ou de enrijecimento.

Os compósitos oferecem vários atrativos, tais como: alta rigidez e resistência específicas, alta resistência à corrosão, bom isolamento térmico e excelente desempenho quando submetidos à condição de fadiga. Os compósitos reforçados por fibras se destacam pelas elevadas relações resistência/peso e rigidez/peso e os materiais com gradação funcional por sua utilização em situações com altos gradientes de temperatura e/ou tensões.

Naturalmente, nem todas as propriedades dos materiais são melhoradas ao mesmo tempo, também não existe normalmente qualquer obrigação nesse sentido. De fato, algumas das propriedades são conflitantes com outras, como isolamento térmico com condutividade térmica. O objetivo de um material compósito é desempenhar apenas as características necessárias para a tarefa a ser realizada.

A natureza heterogênea e complexa da microestrutura dos materiais compósitos apresenta-se como um fator complicador na análise de estruturas por eles constituídas. Levar em conta todos os detalhes microestruturais do material ao longo de uma estrutura real é uma tarefa na maioria das vezes impossível. Além disso, a complexidade do comportamento reológico do material e as limitações de ordem computacional acentuam ainda mais as dificuldades. Uma estratégia para contornar estes obstáculos consiste em se realizar análises em escala macroscópica, onde são empregadas as propriedades efetivas do material homogeneizado [8]. Entretanto a evolução da tecnologia não só beneficiou o surgimento de novos materiais, como também influenciou na melhoria do desempenho dos computadores pessoais. Segundo [2] graças à constante evolução dos computadores pessoais, a aplicação dos métodos numéricos 
na engenharia estrutural vem crescendo vertiginosamente. Há décadas, quando não se dispunha desta importante ferramenta, a análise estrutural era feita de forma bastante simplificada.

Com o aparato computacional disponível atualmente, pode-se desenvolver modelos mais elaborados e próximos do modelo real, minimizando os erros e tornando os resultados mais próximos. Entretanto mesmo que se tenha um supercomputador, se não for escolhido o método numérico coerente para analisar o problema, poderão ocorrer erros numéricos.

O uso de métodos numéricos para a resolução de problemas de engenharia é atualmente imprescindível. Cada vez mais a análise dos problemas de engenharia passa a ser feita através de processos aproximados cujo emprego necessita do computador para permitir a obtenção de respostas confiáveis.

Os primeiros métodos numéricos foram o Método das Diferenças Finitas (MDF), Método dos Elementos Finitos (MEF) e mais recentemente o Método dos Elementos de Contorno (MEC).

\section{ESTRUTURA SANDUÍCHE}

Dentre os materiais compósitos, existem os materiais laminados, que são fabricados por meio da laminação de materiais distintos; a placa laminada é formada por um conjunto de lâminas. Assume-se que estas lâminas estão perfeitamente coladas entre si, ou seja, as lâminas que compõe o laminado comportam-se como uma placa anisotrópica única. Não é admitido deslizamento nem empenamento entre as lâminas; a deformação interfacial é assumida como sendo constante.

Os laminados podem ser constituídos de fibras continuas e unidirecionais, alinhadas paralelamente cada lâmina segundo uma orientação, ou constituídos por dois materiais com propriedades distintas, mais conhecidos como estruturas "sanduíche"; onde um núcleo mais espesso de material menos resistente é coberto com chapas de maiores resistências mecânicas (Figura 1). Estruturas aeronáuticas normalmente adicionam chapas metálicas a núcleos de espumas sólidas. A resistência à corrosão também é uma característica importante no projeto e escolha destes materiais.
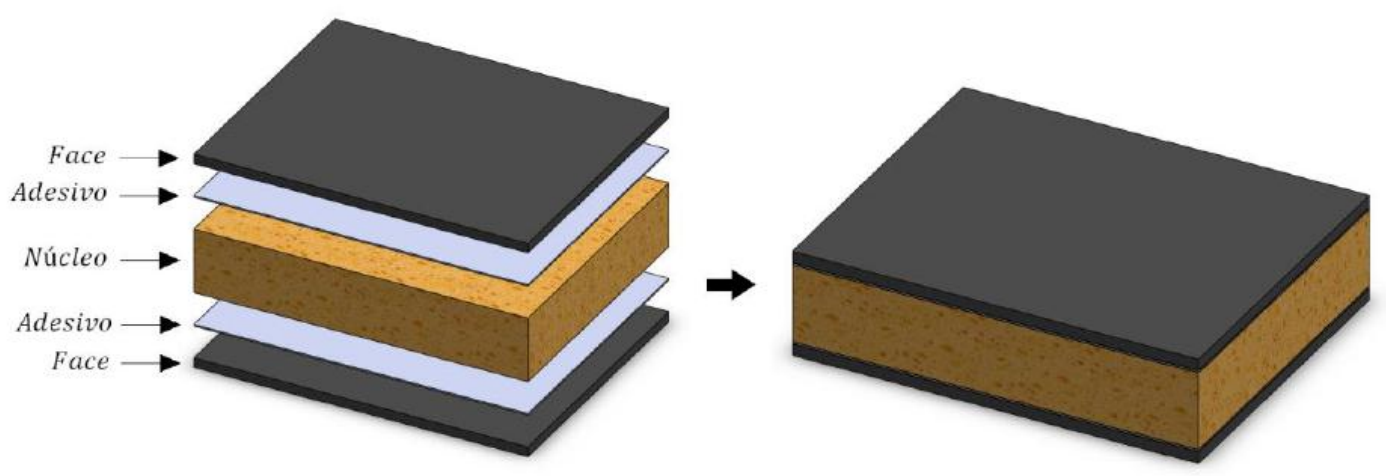

Figura 1. Componentes de uma estrutura sanduíche [4]. 
O princípio da técnica de estruturas do tipo sanduíche consiste em colocar um material leve (geralmente com boas propriedades à compressão) entre duas contra placas com alta rigidez. Este princípio concilia leveza e rigidez à estrutura final. Na Figura 2 são mostrados alguns tipos de estruturas sanduíche.

Segundo [4] as faces têm como principal função suportar as tensões axiais, pelo que é necessário que estas tenham uma elevada rigidez. Aliado a isso, as faces dão à estrutura resistência ao impacto, à corrosão e à umidade.

O núcleo em uma estrutura sanduíche tem como função suportar os esforços de corte e compressão, evitar que as faces deslizem uma relativamente à outra, garantir o melhoramento do comportamento à flexão da estrutura e conferir-lhe um menor peso.

$\mathrm{Na}$ área da construção civil a utilização de estruturas sanduíche ainda é recente. No entanto, para esta área, já existem componentes com características bem desenvolvidas e são utilizados para divisórias, paredes, pisos, forros, e lajes, entre outras aplicações [7].

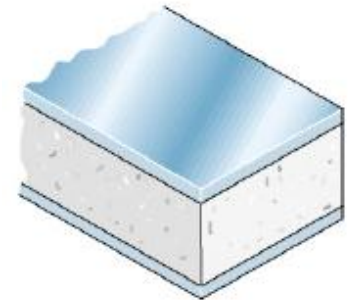

(a)

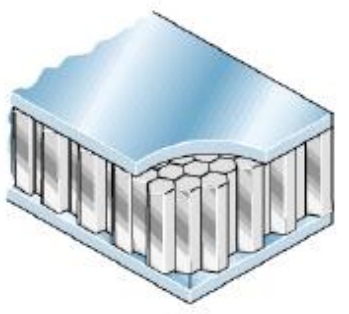

(b)

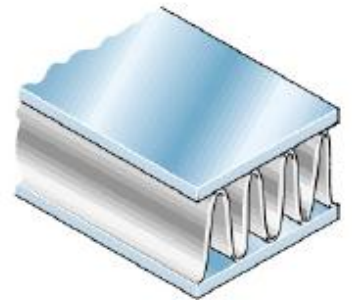

(c)

Figura 2. Estrutura sanduíche com núcleo: sólido (a), em forma de colmeia (b) e corrugado (c) [5].

\section{REGRA DE MISTURA}

A regra de mistura é o método mais simples para se obter as propriedades mecânicas de um material heterogêneo. Na equação (1) encontra-se a forma genérica da formulação da regra de mistura.

$$
P_{h}=P_{m} f_{m}+P_{i} f_{i}
$$

onde $P_{h}, P_{m}$ e $P_{i}$ representam a propriedade desejada do material compósito, a propriedade da matriz e a propriedade da inclusão, respectivamente; e $f_{m}$ e $f_{i}$ representam respectivamente a fração volumétrica da matriz e da inclusão.

A regra de mistura pode ser usada para encontrar o módulo de elasticidade, coeficiente de Poisson, condutividade térmica, viscosidade, entre outras propriedades [10].

As propriedades mecânicas dos materiais compósitos são determinadas pelas propriedades dos materiais que o constituem e também depende da direção do carregamento aplicado [9]. Na seção seguinte é apresentada a dedução para se encontrar o módulo de elasticidade em um material compósito reforçado por fibra, onde existem apenas forças no estado uniaxial, e o carregamento está distribuído transversalmente à direção das fibras. 


\subsection{Carregamento transversal às fibras}

Assumindo que o eixo de carga está orientado perpendicularmente à direção das fibras e que as fibras são placas ao longo de toda a seção transversal do material (Figura 3); para este caso, devem existir equilíbrios de forças para manter vinculada cada junção da fibra com a matriz, sendo assim:

$$
\sigma_{f}=\sigma_{m}
$$

Porém como fibra e matriz são materiais diferentes, com diferentes módulos de elasticidade, a resistência à deformação será diferente; portanto $\varepsilon_{f} \neq \varepsilon_{m}$.

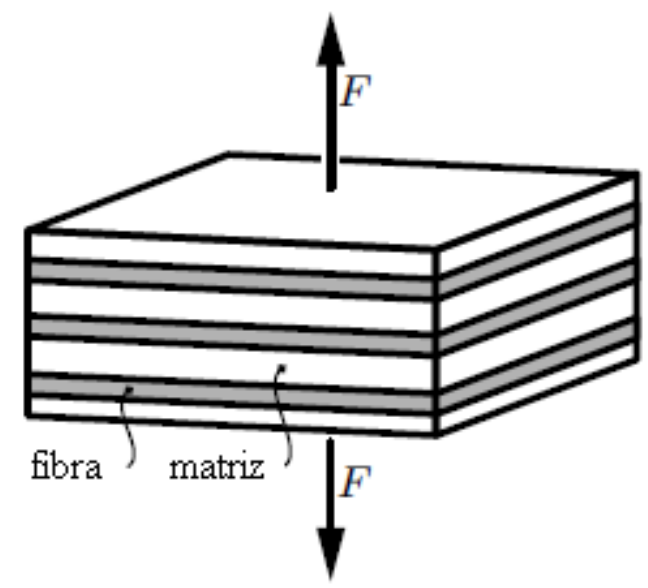

Figura 3. Compósito reforçado por fibra com carga na direção ortogonal às fibras [9].

O comprimento total do material é igual à soma dos comprimentos da fibra e da matriz:

$$
\mathrm{l}=\mathrm{l}_{\mathrm{f}}+\mathrm{l}_{\mathrm{m}}
$$

A partir da equação 3 e da Lei de Hooke, encontra-se o módulo de elasticidade transversal.

$$
E_{\perp}=\frac{E_{m}}{1+f_{f}\left(\frac{E_{m}}{E_{f}}-1\right)} .
$$

A regra de mistura é apenas um modelo aproximado que não leva em conta as tensões localizadas. Segundo [9], é mais adequado usar a regra de mistura para determinar o módulo de elasticidade do que para determinar o coeficiente de Poisson. Isto ocorre devido à elevada ordem de grandeza do módulo de elasticidade, tornando-o menos sensível a tensões residuais resultantes, tais como as tensões decorrentes da adição de fibras à matriz. 


\section{MÉTODO DOS ELEMENTOS DE CONTORNO}

Uma das principais vantagens do Método dos Elementos de Contorno (MEC) é que, para o problema em estudo, não é preciso dividir o domínio em subdomínios, sendo necessário apenas discretizar o contorno da região considerada.

O Método dos Elementos de Contorno pode ser facilmente ajustável a contornos geometricamente complexos [3]. Além do mais, uma vez que todas as aproximações estão restritas ao contorno, pode-se modelar regiões com gradientes elevados com melhor exatidão em relação ao Método dos Elementos Finitos (MEF).

Em comparação com o MEF, o MEC apresenta as seguintes principais vantagens: modelagem própria para domínios infinitos, análise de problemas com singularidade, grande redução do número de equações e volume de dados (principalmente quando se trata de meios tridimensionais infinitos como o solo) e a inexistência de erros de interpolação no domínio para problemas lineares. Em contrapartida, o Método dos Elementos de Contorno não apresenta características vantajosas relevantes no que diz respeito à análise de estruturas reticuladas e cascas gerais, quando comparado ao MEF. Com isso, em [1], tira-se proveito das melhores características dos dois métodos, realizando análises da interação solo-estrutura, usando o acoplamento entre o MEC e o MEF, onde o MEF é usado para a estrutura (pórticos e cascas) e o MEC para o solo ou semi-espaço infinito.

A discussão sobre o método mais adequado deve levar em consideração o tipo de análise. Portanto, cabe ao engenheiro conhecer as potencialidades e limitações de cada método para escolher o mais apropriado para a simulação.

\section{ANÁLISES}

\subsection{Estudo de propriedades mecânicas efetivas de compósitos sanduíche}

O uso de estruturas do tipo sanduíche surge da necessidade de aumentar a eficiência das estruturas ao combinar um peso muito menor aliado a uma grande resistência mecânica [4]. Isto é conseguido porque as duas faces são mantidas a uma distância elevada uma da outra, com o intuito de aumentar o momento de inércia, uma vez que este é proporcional ao cubo da altura da viga ou painel. Como consequência, também a resistência à flexão da estrutura aumenta, conferindo assim esta tão desejada propriedade. Por outro lado, e em termos de peso, o ganho é conseguido através do núcleo, porque o uso de materiais de elevada resistência faz sentido apenas nas faces, onde as tensões normais máximas, devidas à flexão, ocorrem, e porque são as zonas onde a estrutura está sujeita às forças axiais. A seguir são mostradas algumas aplicações envolvendo placas sanduíche.

Todas as aplicações de placas sanduíche tiveram suas lâminas modeladas da seguinte maneira.

Primeiramente, foram geradas as malhas de elementos de contorno. Para isso todas as arestas das lâminas foram divididas em dez subdivisões iguais nas direções $\mathrm{x}$ e y, e na direção z tiveram quatro subdivisões, como mostra a Figura 4. 


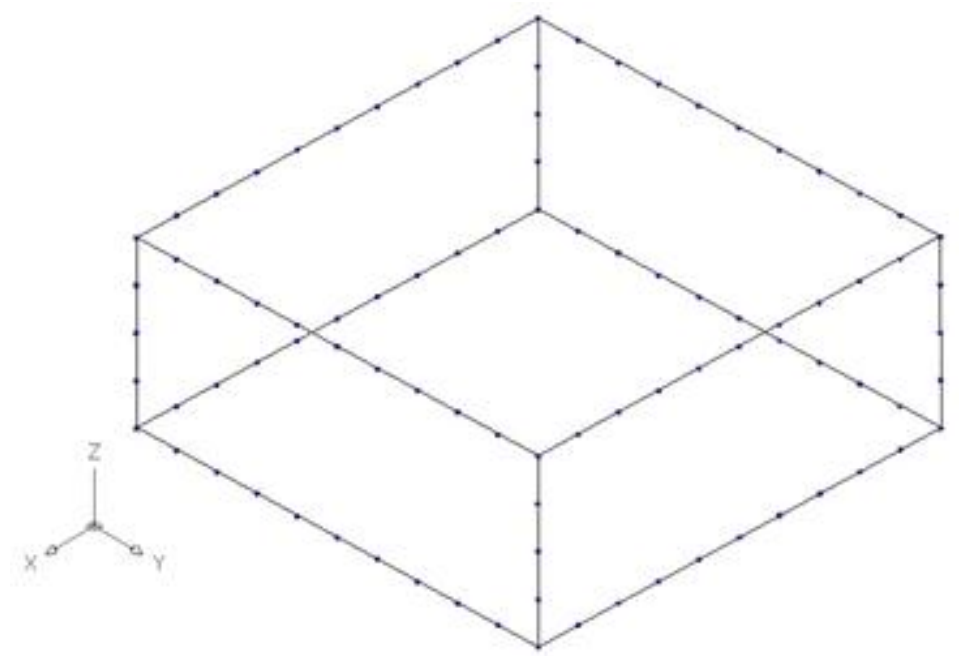

Figura 4. Subdivisões das arestas da lâmina.

Com as arestas subdivididas, partiu-se para a subdivisão da placa em elementos de contorno. Todo contorno foi dividido em elementos triangulares com três nós, como está representado na Figura 5. As placas foram elaboradas desta forma porque as dimensões em x e y têm ordem de grandeza maior que em z. Além disso, ao modificar as dimensões das placas o número de elementos usados seria o mesmo.

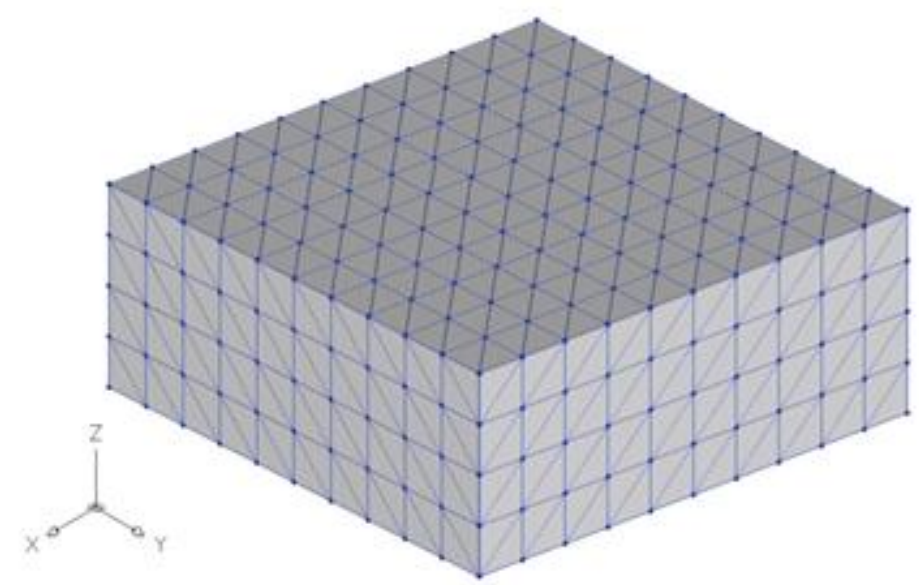

Figura 5. Subdivisão da lâmina em elementos de contorno triangulares.

Como já foi dito nos capítulos anteriores os compósitos sanduíche são constituídos de três placas: uma face superior, o núcleo e a face inferior, onde o material da face superior pode ser ou não igual à face inferior. Para realizar a ligação entre as placas no programa de análise utilizado [1], foi feito o acoplamento entre os nós por meio da técnica de sub-regiões. Acoplando os graus de liberdade de cada nó presente na região de ligação, admitindo que as faces estejam perfeitamente aderidas ao núcleo.

Admitiu-se para todas as aplicações que os materiais e as espessuras das faces são iguais. A estrutura sanduíche está apresentada na Figura 6, onde a largura é igual ao comprimento e h1 e h2 representam as espessuras das faces e do núcleo respectivamente. A figura 7 ilustra os apoios e carregamentos utilizados. 


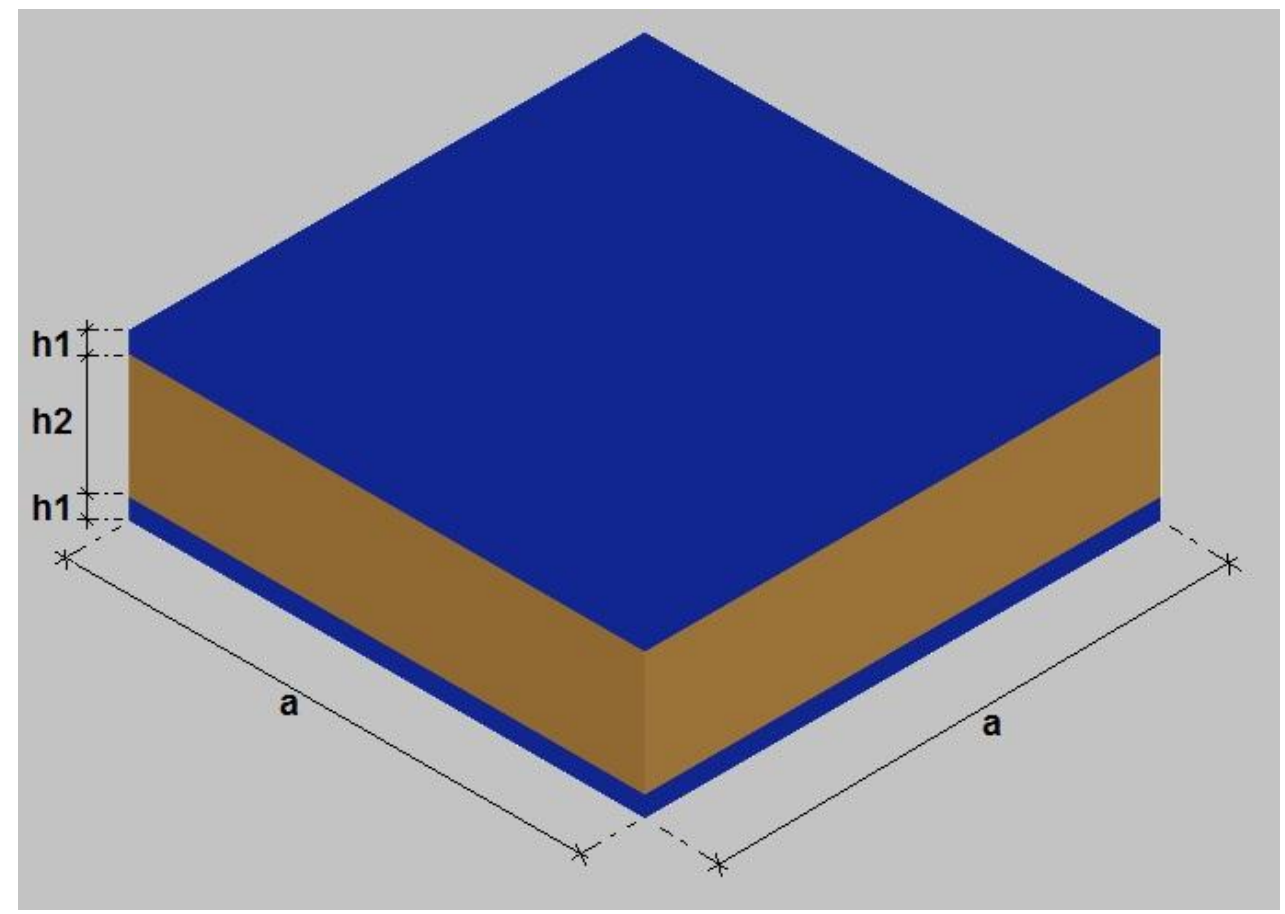

Figura 6. Dimensões das estruturas sanduíche.

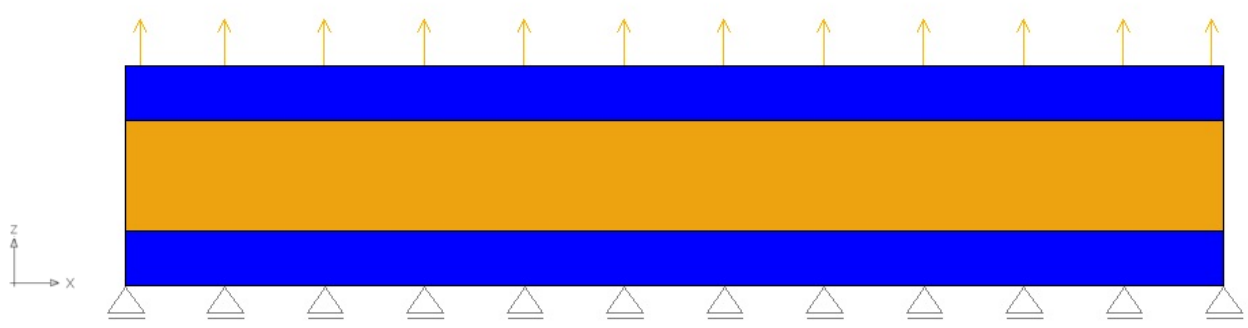

Figura 7. Apoios e carregamentos.

\subsection{Exemplo 1: estudo das propriedades mecânicas efetivas de estruturas sanduíche}

Neste exemplo, pretende-se obter as propriedades mecânicas efetivas dos compósitos sanduíche através do MEC e fazer uma comparação com a resposta obtida pela regra de mistura.

Uma placa quadrada, simplesmente apoiada é analisada para diferentes materiais e espessuras. A placa está submetida a uma tensão de tração transversal uniforme de $1 \mathrm{MPa}$. Os materiais usados para as faces foram o aço e o alumínio; e para o núcleo, espuma de alumínio (alumínio light) e espuma de poliuretano (PU). A Tabela 1 mostra a composição dos materiais estudados e na Tabela 2 estão as propriedades mecânicas dos mesmos.

Tabela 1. Composição dos materiais compósitos estudados.

\begin{tabular}{c}
\hline Face - Núcleo - Face \\
\hline Aço - Alumínio Light - Aço \\
Aço - PU - Aço \\
Alumínio - Alumínio Light - Alumínio \\
Alumínio - PU - Alumínio \\
\hline
\end{tabular}


Tabela 2. Propriedades dos materiais.

\begin{tabular}{cccc}
\hline & $\boldsymbol{\rho}\left(\mathbf{K g} / \mathbf{m}^{\mathbf{3}}\right)$ & $\mathbf{E}(\mathbf{M p a})$ & $\boldsymbol{v}$ \\
\hline Alumínio Light & 500 & 5000 & 0,33 \\
Poliuretano & 1150 & 1164 & 0,36 \\
Alumínio & 2700 & 69000 & 0,33 \\
Aço & 7800 & 210000 & 0,3 \\
\hline
\end{tabular}

Para a realização deste estudo foram modeladas três placas sanduíche com as dimensões apresentadas na Tabela 3:

Tabela 3. Dimensões das placas sanduíche.

\begin{tabular}{cccc}
\hline & $\boldsymbol{\rho}\left(\mathbf{K g} / \mathbf{m}^{\mathbf{3}}\right)$ & $\mathbf{E}(\mathbf{M p a})$ & $\boldsymbol{v}$ \\
\hline Alumínio Light & 500 & 5000 & 0,33 \\
Poliuretano & 1150 & 1164 & 0,36 \\
Alumínio & 2700 & 69000 & 0,33 \\
Aço & 7800 & 210000 & 0,3 \\
\hline
\end{tabular}

A relação espessura/largura utilizada para a placa sanduíche $S 1$ nesse exemplo foi a mesma usada em [6].

Para a validação do modelo, tomou-se a placa sanduíche com as três lâminas do mesmo material, ou seja, uma placa homogênea. Com os deslocamentos fornecidos pelo programa computacional, calculou-se o módulo de elasticidade e o coeficiente de Poisson e comparouse com os valores fornecidos pela literatura. Ver Tabelas 4 a 9.

Tabela 4. Placa de aço com dimensões correspondentes às da placa sanduíche S1.

\begin{tabular}{c|c|c|c|c|c|c|c|c|c}
\cline { 2 - 9 } & \multicolumn{4}{c|}{ Valores fornecidos pelo MEC } & \multicolumn{2}{c|}{ Literatura } & \multicolumn{2}{c}{ Erro } \\
\hline Direção & $\Delta \mathrm{L}(\mathrm{m})$ & $\mathrm{L}(\mathrm{m})$ & $\varepsilon$ & $\mathrm{E}$ & $v$ & $\mathrm{E}$ & $v$ & $\mathrm{E}$ & $v$ \\
\hline $\mathrm{x}$ & $-1,43 \mathrm{E}-06$ & 1 & $-1,43 \mathrm{E}-06$ & & & & & & \\
$\mathrm{y}$ & $-1,43 \mathrm{E}-06$ & 1 & $-1,43 \mathrm{E}-06$ & 209997 & 0,30 & 210000 & 0,30 & $0,002 \%$ & $0,00 \%$ \\
$\mathrm{z}$ & $1,52 \mathrm{E}-06$ & 0,32 & $4,76 \mathrm{E}-06$ & & & & & & \\
\hline
\end{tabular}

Tabela 5. Placa de alumínio com dimensões correspondentes às da placa sanduíche S1.

\begin{tabular}{c|c|c|c|c|c|c|c|c|c}
\cline { 2 - 9 } & \multicolumn{4}{c|}{ Valores fornecidos pelo MEC } & \multicolumn{2}{c|}{ Literatura } & \multicolumn{2}{c}{ Erro } \\
\hline Direção & $\Delta \mathrm{L}(\mathrm{m})$ & $\mathrm{L}(\mathrm{m})$ & $\varepsilon$ & $\mathrm{E}$ & $v$ & $\mathrm{E}$ & $v$ & $\mathrm{E}$ & $v$ \\
\hline $\mathrm{x}$ & $-4,78 \mathrm{E}-06$ & 1 & $-4,78 \mathrm{E}-06$ & & & & & & \\
$\mathrm{y}$ & $-4,78 \mathrm{E}-06$ & 1 & $-4,78 \mathrm{E}-06$ & 68999 & 0,33 & 69000 & 0,33 & $0,002 \%$ & $0,00 \%$ \\
$\mathrm{z}$ & $4,64 \mathrm{E}-06$ & 0,32 & $1,45 \mathrm{E}-05$ & & & & & & \\
\hline
\end{tabular}

Tabela 6. Placa de aço com dimensões correspondentes às da placa sanduíche S2.

\begin{tabular}{c|c|c|c|c|c|c|c|c|c}
\cline { 2 - 9 } & \multicolumn{4}{c|}{ Valores fornecidos pelo MEC } & \multicolumn{2}{c|}{ Literatura } & \multicolumn{2}{c}{ Erro } \\
\hline Direção & $\Delta \mathrm{L}(\mathrm{m})$ & $\mathrm{L}(\mathrm{m})$ & $\varepsilon$ & $\mathrm{E}$ & $v$ & $\mathrm{E}$ & $v$ & $\mathrm{E}$ & $v$ \\
\hline $\mathrm{x}$ & $-1,43 \mathrm{E}-06$ & 1 & $-1,43 \mathrm{E}-06$ & & & & & & \\
$\mathrm{y}$ & $-1,43 \mathrm{E}-06$ & 1 & $-1,43 \mathrm{E}-06$ & 209993 & 0,30 & 210000 & 0,30 & $0,003 \%$ & $0,00 \%$ \\
$\mathrm{z}$ & $9,52 \mathrm{E}-07$ & 0,2 & $4,76 \mathrm{E}-06$ & & & & & & \\
\hline
\end{tabular}


Tabela 7. Placa de alumínio com dimensões correspondentes às da placa sanduíche S2.

\begin{tabular}{c|c|c|c|c|c|c|c|c|c}
\cline { 2 - 9 } & \multicolumn{4}{c|}{ Valores fornecidos pelo MEC } & \multicolumn{2}{c|}{ Literatura } & \multicolumn{2}{c}{ Erro } \\
\hline Direção & $\Delta \mathrm{L}(\mathrm{m})$ & $\mathrm{L}(\mathrm{m})$ & $\varepsilon$ & $\mathrm{E}$ & $v$ & $\mathrm{E}$ & $v$ & $\mathrm{E}$ & $v$ \\
\hline $\mathrm{x}$ & $-4,78 \mathrm{E}-06$ & 1 & $-4,78 \mathrm{E}-06$ & & & & & & \\
$\mathrm{y}$ & $-4,78 \mathrm{E}-06$ & 1 & $-4,78 \mathrm{E}-06$ & 68998 & 0,33 & 69000 & 0,33 & $0,004 \%$ & $0,00 \%$ \\
$\mathrm{z}$ & $2,90 \mathrm{E}-06$ & 0,2 & $1,45 \mathrm{E}-05$ & & & & & & \\
\hline
\end{tabular}

Tabela 8. Placa de aço com dimensões correspondentes às da placa sanduíche S3.

\begin{tabular}{c|c|c|c|c|c|c|c|c|c}
\cline { 2 - 9 } & \multicolumn{4}{c|}{ Valores fornecidos pelo MEC } & \multicolumn{2}{c|}{ Literatura } & \multicolumn{2}{c}{ Erro } \\
\hline Direção & $\Delta \mathrm{L}(\mathrm{m})$ & $\mathrm{L}(\mathrm{m})$ & $\varepsilon$ & $\mathrm{E}$ & $v$ & $\mathrm{E}$ & $v$ & $\mathrm{E}$ & $v$ \\
\hline $\mathrm{X}$ & $-1,43 \mathrm{E}-06$ & 1 & $-1,43 \mathrm{E}-06$ & & & & & & \\
$\mathrm{Y}$ & $-1,43 \mathrm{E}-06$ & 1 & $-1,43 \mathrm{E}-06$ & 210396 & 0,30 & 210000 & 0,30 & $0,19 \%$ & $0,00 \%$ \\
$\mathrm{Z}$ & $9,51 \mathrm{E}-07$ & 0,2 & $4,75 \mathrm{E}-06$ & & & & & & \\
\hline
\end{tabular}

Tabela 9. Placa de alumínio com dimensões correspondentes às da placa sanduíche S3.

\begin{tabular}{c|c|c|c|c|c|c|c|c|c}
\cline { 2 - 9 } & \multicolumn{4}{c|}{ Valores fornecidos pelo MEC } & \multicolumn{2}{c|}{ Literatura } & \multicolumn{2}{c}{ Erro } \\
\hline Direção & $\Delta \mathrm{L}(\mathrm{m})$ & $\mathrm{L}(\mathrm{m})$ & $\varepsilon$ & $\mathrm{E}$ & $v$ & $\mathrm{E}$ & $v$ & $\mathrm{E}$ & $v$ \\
\hline $\mathrm{x}$ & $-4,78 \mathrm{E}-06$ & 1 & $-4,78 \mathrm{E}-06$ & & & & & & \\
$\mathrm{y}$ & $-4,78 \mathrm{E}-06$ & 1 & $-4,78 \mathrm{E}-06$ & 69135 & 0,33 & 69000 & 0,33 & $0,20 \%$ & $0,00 \%$ \\
$\mathrm{z}$ & $2,89 \mathrm{E}-06$ & 0,2 & $1,45 \mathrm{E}-05$ & & & & & & \\
\hline
\end{tabular}

Como pode ser notado, os resultados obtidos pelo acoplamento MEC/MEC apresentam um erro muito pequeno em relação aos valores da literatura.

Em seguida, mudou-se apenas as propriedades do material do núcleo e calculou-se as propriedades mecânicas efetivas dos compósitos. Na Figura 8 está representada a deformada no plano XZ, e, para uma melhor visualização, esta foi ampliada.

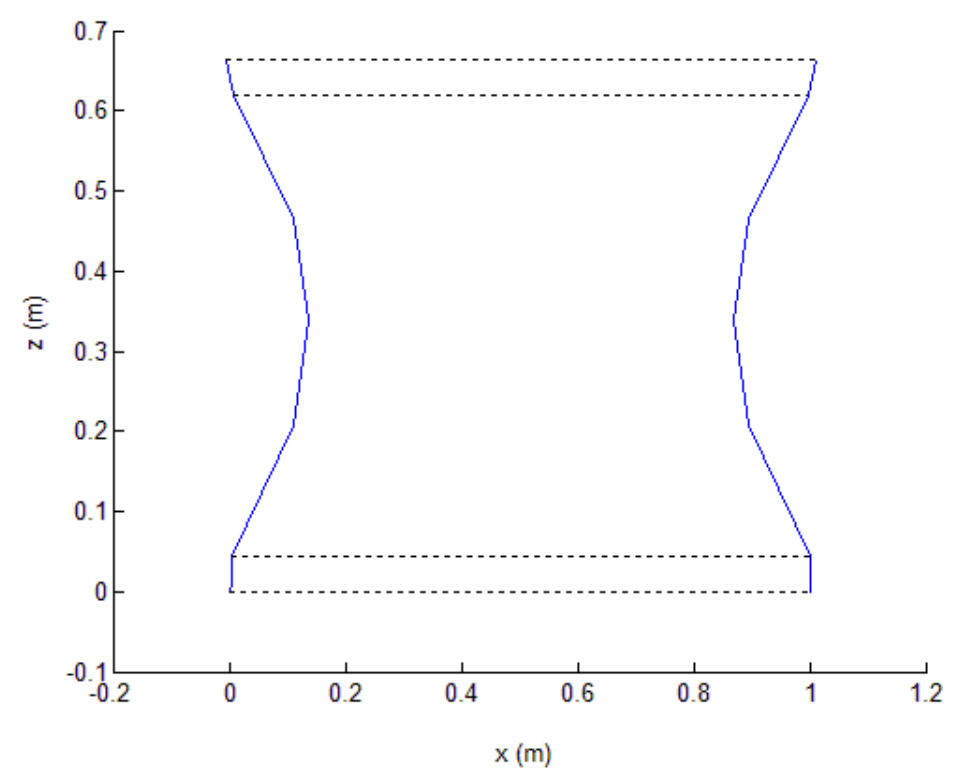

Figura 8. Deformada da estrutura sanduíche. 
Como se pode ver na Figura 8, a deformação varia ao longo da espessura, portanto para se determinar o coeficiente de Poisson efetivo, fez-se a média do deslocamento total nas faces. Nas tabelas 10 a 12 estão apresentadas as propriedades mecânicas efetivas, módulo de elasticidade e coeficiente de Poisson, dos materiais compósitos estudados e a diferença entre os módulos de elasticidade obtidos pelo MEC e pela regra de mistura.

A regra de mistura apresentada na equação (4) foi deduzida partindo do pressuposto que as fibras são placas ao longo de toda a seção transversal do material, consequentemente usouse esta equação para obtenção do módulo de elasticidade e como parâmetro de comparação.

Tabela 10. Propriedades mecânicas da placa sanduíche S1.

\begin{tabular}{ccccc}
\hline & E (Mpa) & $\boldsymbol{v}$ & Regra de Mistura & Erro \\
\hline Aço - Al Light - Aço & 8783 & 0,04 & 6614,17 & $32,79 \%$ \\
Aço - PU - Aço & 2289 & 0,01 & 1549,14 & $47,75 \%$ \\
Al - Al Light - Al & 8146 & 0,10 & 6509,43 & $25,13 \%$ \\
Al - PU - Al & 2229 & 0,03 & 1543,32 & $44,40 \%$ \\
\hline
\end{tabular}

Tabela 11. Propriedades mecânicas da placa sanduíche S2.

\begin{tabular}{ccccc}
\hline & E (Mpa) & $\boldsymbol{v}$ & Regra de Mistura & Erro \\
\hline Aço - Al Light - Aço & 11548 & 0,04 & 8203,13 & $40,78 \%$ \\
Aço - PU - Aço & 3096 & 0,01 & 1932,86 & $60,17 \%$ \\
Al - Al Light - Al & 10486 & 0,10 & 7949,31 & $31,91 \%$ \\
Al - PU - Al & 3002 & 0,03 & 1918,42 & $56,50 \%$ \\
\hline
\end{tabular}

Tabela 12. Propriedades mecânicas da placa sanduíche S3.

\begin{tabular}{ccccc}
\hline & E (Mpa) & $\boldsymbol{v}$ & Regra de Mistura & Erro \\
\hline Aço - Al Light - Aço & 7317 & 0,07 & 5540,90 & $32,06 \%$ \\
Aço - PU - Aço & 1947 & 0,02 & 1292,54 & $50,60 \%$ \\
Al - Al Light - Al & 6712 & 0,16 & 5511,18 & $21,79 \%$ \\
Al - PU - Al & 1869 & 0,06 & 1290,91 & $44,79 \%$ \\
\hline
\end{tabular}

Constatou-se que para todos os casos analisados, o erro percentual entre os métodos foi elevado, sendo alguns acima de 50\%. Este fato pode ser atribuído à simplicidade da formulação apresentada em [9]. Percebe-se que em nenhum momento a equação (4) leva em consideração os coeficientes de Poisson dos materiais constituintes, ou seja, ela assume que as deformações derivadas do efeito de Poisson são constantes ao longo de todo o material. Entretanto as análises realizadas foram em três dimensões e admitiu-se um acoplamento das subregiões nas três direções, ou seja, uma colagem perfeita entre as placas. Com isso, quando se tem materiais com diferentes coeficientes de Poisson ligados e esses materiais estão submetidos a um carregamento os mesmos se deformam de diferentes formas, como se pode observar na Figura 8.

Essa deformação, resultante do efeito Poisson, acarreta o surgimento de uma energia, proveniente das tensões paralelas à direção das placas nas regiões de ligação. Devido a essas tensões, a rigidez efetiva determinada pelo MEC é maior quando comparada à regra de mistura. 


\subsection{Exemplo 2: Análise da relação rigidez/peso de estruturas sanduíche}

Nesta aplicação, busca-se determinar a relação rigidez/peso da estrutura sanduíche e compará-la com uma placa homogênea constituída pelo material da face que tenha a mesma relação rigidez/peso.

A placa sanduíche foi submetida às mesmas condições de apoio e carregamento do exemplo anterior, e utilizou-se os mesmos materiais. Ver Tabelas 1 e 2.

Para este exemplo, manteve-se a espessura das faces em $4 \mathrm{~cm}$ e variou-se as alturas do núcleo de $12 \mathrm{em} 12 \mathrm{~cm}$, partindo-se de $12 \mathrm{~cm}$ até $60 \mathrm{~cm}$, como é mostrado na Tabela 13.

Tabela 13. Espessuras dos núcleos usados no exemplo 2 e sua respectiva espessura total.

\begin{tabular}{lcc}
\hline & $\mathbf{h}_{\mathbf{2}}(\mathbf{c m})$ & $\mathbf{h}_{\text {total }}(\mathbf{c m})$ \\
\hline $\mathbf{S 1}$ & 12 & 20 \\
$\mathbf{S 2}$ & 24 & 32 \\
$\mathbf{S 3}$ & 36 & 44 \\
$\mathbf{S 4}$ & 48 & 56 \\
$\mathbf{S 5}$ & 60 & 68 \\
\hline
\end{tabular}

Em seguida, calculou-se as propriedades mecânicas efetivas dos compósitos sanduíches pelo MEC e as suas respectivas rigidezes à flexão (equação 5). Com as densidades dos materiais fornecidas pela Tabela 2, obteve-se os pesos e consequentemente a relação rigidez/peso pela equação 6. Ver tabelas 14 a 17.

$$
D=\frac{E \cdot h^{3}}{12 \cdot\left(1-v^{2}\right)}
$$

onde $\mathrm{D}, \mathrm{E}, \mathrm{h}$ e $v$ são rigidez à flexão, módulo de elasticidade, altura total do compósito e coeficiente de Poisson, respectivamente. E

$$
\frac{D}{P}=\frac{E \cdot h^{2}}{12 \cdot\left(1-v^{2}\right) \cdot \rho \cdot a^{2} \cdot g}
$$

onde $\mathrm{D} / \mathrm{P}, \rho$ e g são rigidez/peso, densidade e aceleração da gravidade, respectivamente.

Tabela 14. Propriedades mecânicas efetivas para a placa aço - alumínio light - aço.

\begin{tabular}{ccccc}
\hline \multicolumn{5}{c}{ Aço - Al Light - Aço } \\
\hline & E (Mpa) & $v$ & D (N.m) & D/P (m) \\
S1 & 11548,00 & 0,04 & $7,71 \mathrm{E}+06$ & 1141,05 \\
S2 & 8783,00 & 0,04 & $2,40 \mathrm{E}+07$ & 3270,30 \\
S3 & 7699,45 & 0,04 & $5,47 \mathrm{E}+07$ & 6899,96 \\
S4 & 7082,21 & 0,04 & $1,04 \mathrm{E}+08$ & 12179,64 \\
S5 & 6684,24 & 0,04 & $1,75 \mathrm{E}+08$ & 19249,18 \\
\hline
\end{tabular}


Tabela 15. Propriedades mecânicas efetivas para a placa aço - PU - aço.

\begin{tabular}{ccccc}
\hline \multicolumn{5}{c}{ Aço -PU - Aço } \\
\hline & E (Mpa) & $v$ & D (N.m) & D/P (m) \\
S1 & 3095,92 & 0,01 & $2,06 E+06$ & 274,41 \\
S2 & 2288,91 & 0,01 & $6,25 E+06$ & 704,27 \\
S3 & 1970,84 & 0,01 & $1,40 E+07$ & 1367,78 \\
S4 & 1790,26 & 0,01 & $2,62 E+07$ & 2262,08 \\
S5 & 1674,75 & 0,01 & $4,39 E+07$ & 3392,31 \\
\hline
\end{tabular}

Tabela 16. Propriedades mecânicas efetivas para a placa alumínio - alumínio light - alumínio.

\begin{tabular}{ccccc}
\hline \multicolumn{5}{c}{ Al - Al Light - Al } \\
\hline & E (Mpa) & $v$ & D (N.m) & D/P (m) \\
S1 & 10485,6 & 0,10 & $7,07 E+06$ & 2609,56 \\
S2 & 8145,55 & 0,10 & $2,25 E+07$ & 6822,32 \\
S3 & 7220,00 & 0,10 & $5,18 \mathrm{E}+07$ & 13336,2 \\
S4 & 6700,82 & 0,10 & $9,91 \mathrm{E}+07$ & 22143,2 \\
S5 & 6369,43 & 0,10 & $1,68 \mathrm{E}+08$ & 33273,9 \\
\hline
\end{tabular}

Tabela 17. Propriedades mecânicas efetivas para a placa alumínio - PU - alumínio.

\begin{tabular}{ccccc}
\hline \multicolumn{5}{c}{ Al - PU - Al } \\
\hline & E (Mpa) & $v$ & D (N.m) & D/P (m) \\
S1 & 3002,32 & 0,03 & $2,00 E+06$ & 577,01 \\
S2 & 2228,62 & 0,03 & $6,09 \mathrm{E}+06$ & 1262,35 \\
S3 & 1919,56 & 0,03 & $1,36 \mathrm{E}+07$ & 2207,31 \\
S4 & 1742,84 & 0,03 & $2,55 \mathrm{E}+07$ & 3388,89 \\
S5 & 1629,97 & 0,03 & $4,27 \mathrm{E}+07$ & 4809,73 \\
\hline
\end{tabular}

Em seguida, calculou-se a espessura para que a placa homogênea tenha a rigidez equivalente ao compósito. Encontrou-se também a espessura da placa homogênea para que ela tenha a mesma relação rigidez/peso que o material compósito. Isto foi feito para todos os casos de materiais e espessuras.

Com os dados obtidos, plotou-se os gráficos representados nas Figuras 11 e 12. Em azul, estão os dados referentes à placa com a mesma relação rigidez/peso que o material compósito; e em vermelho, com a mesma rigidez que o compósito. 


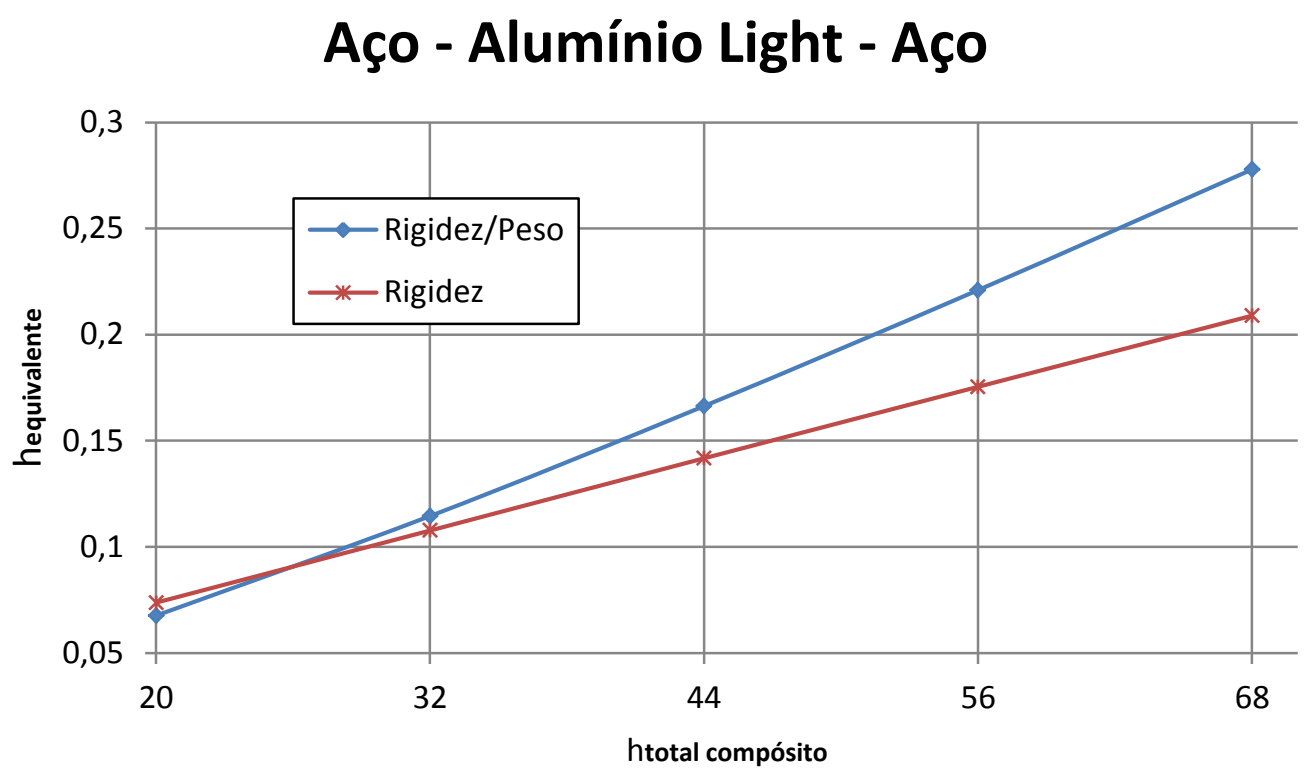

Figura 9. Espessura total do compósito pela espessura equivalente da placa homogênea de aço, para o material composto por aço - alumínio light - aço.

\section{Aço - PU - Aço}

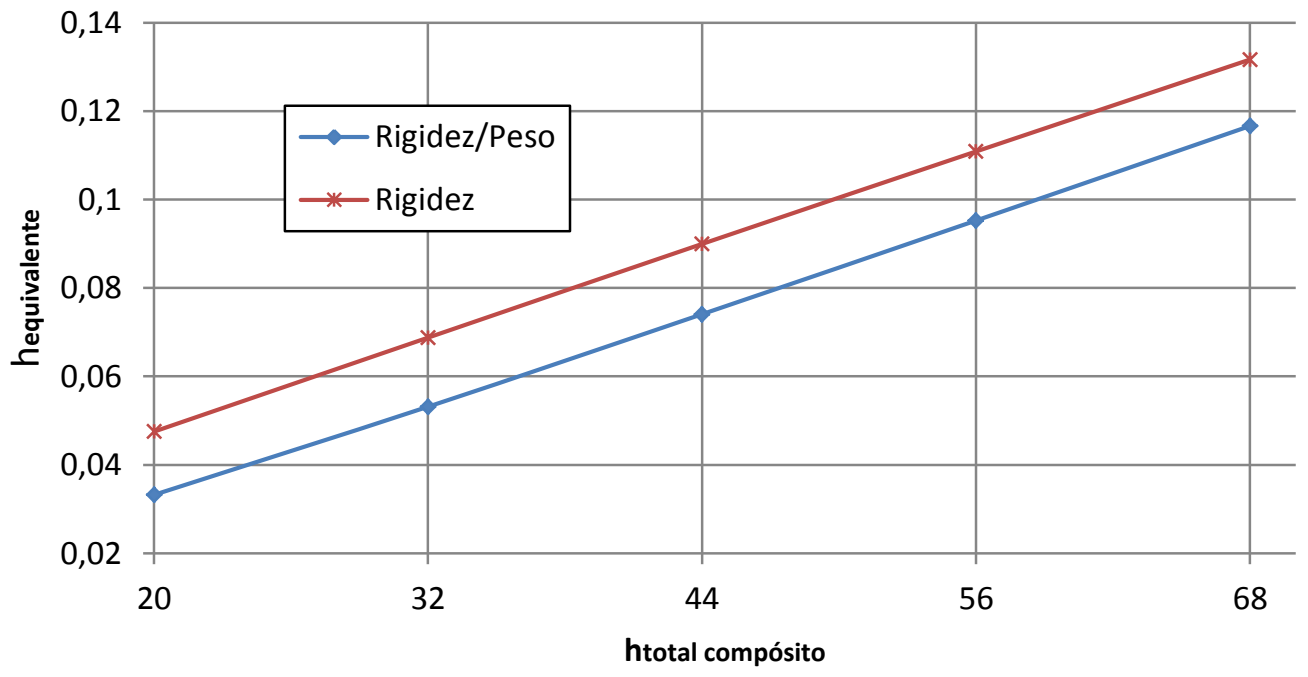

Figura 10. Espessura total do compósito pela espessura equivalente da placa homogênea de aço, para o material composto por aço - PU - aço. 


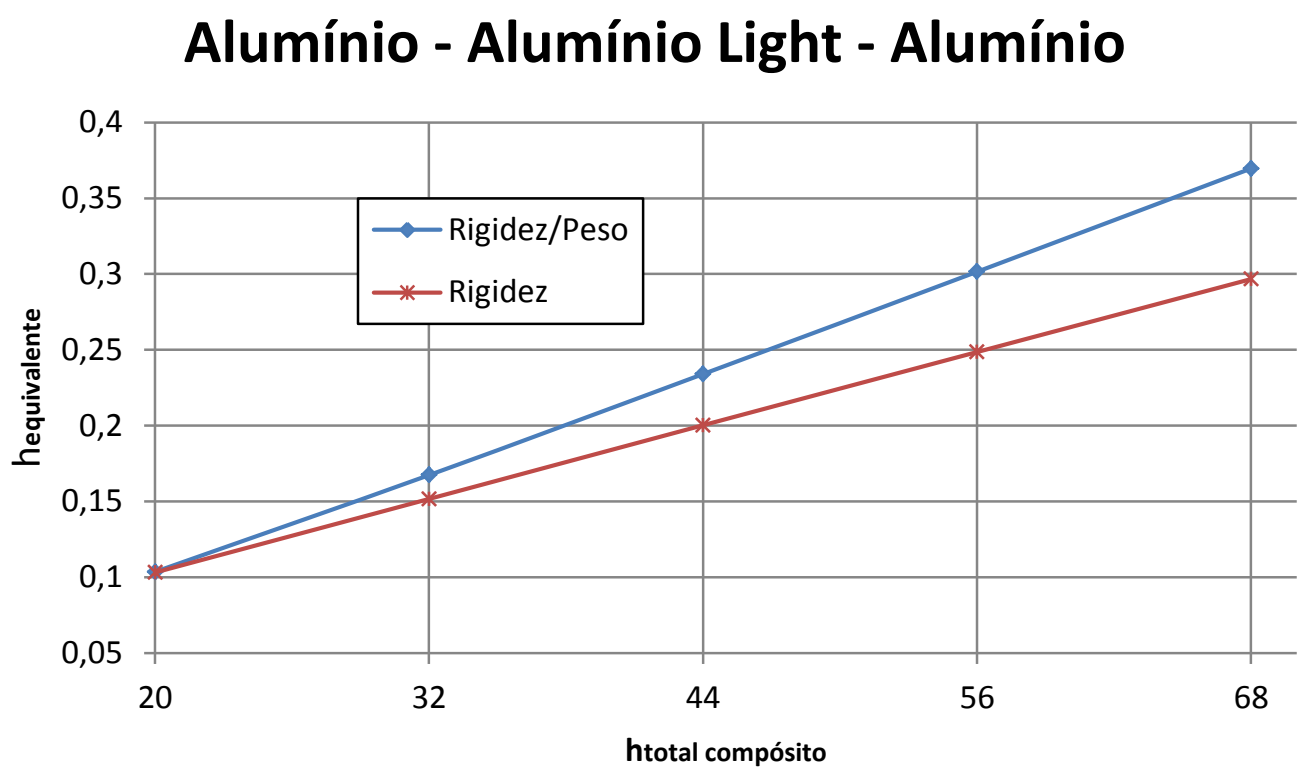

Figura 11. Espessura total do compósito pela espessura equivalente da placa homogênea de alumínio, para o material composto por alumínio - alumínio light - alumínio.

\section{Alumínio - PU - Alumínio}

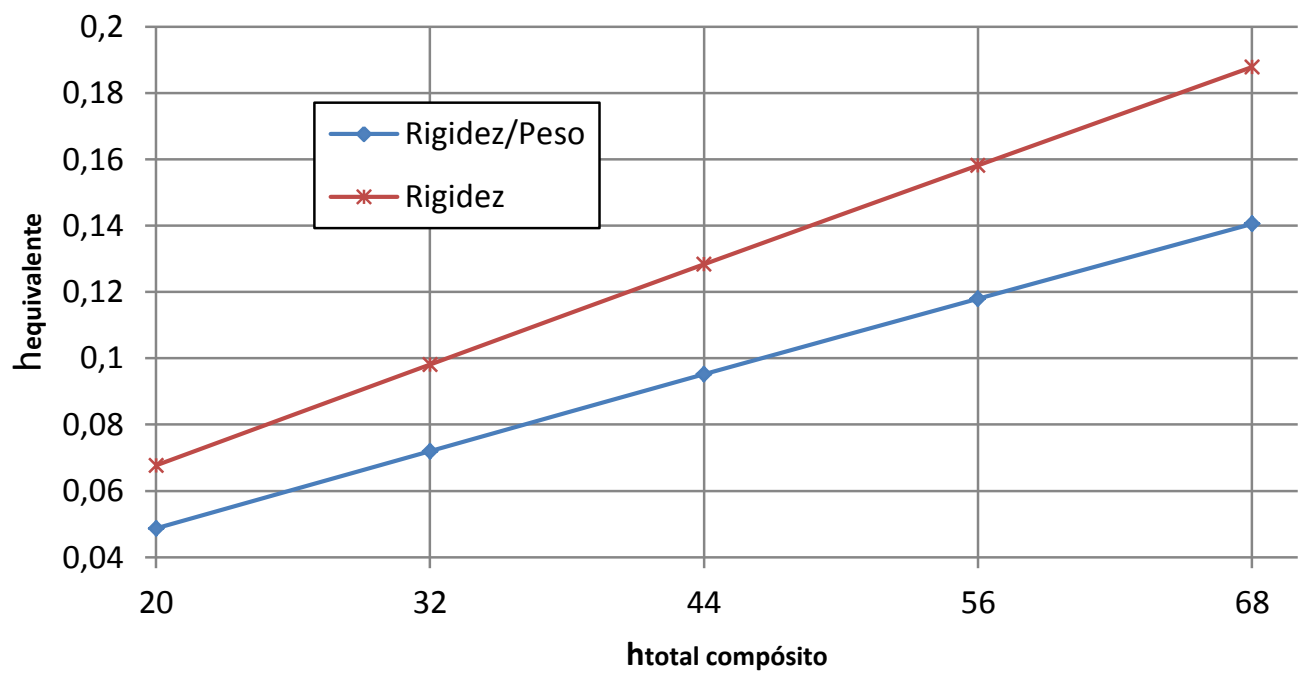

Figura 12. Espessura total do compósito pela espessura equivalente da placa homogênea de alumínio, para o material composto por alumínio - PU - alumínio.

Avaliando os resultados obtidos, observa-se que: se os pontos da curva de rigidez estiverem abaixo da curva rigidez/peso, o material compósito tem uma melhor relação rigidez/peso que uma chapa homogênea de mesma rigidez; caso contrário, a placa homogênea de mesma rigidez tem uma melhor relação rigidez/peso.

Além disso, em todos os casos, a espessura do material homogêneo foi menor que a espessura do material compósito. Entretanto, ao se analisar os materiais com relação a sua rigidez/peso, os materiais constituídos com espuma metálica (alumínio light) apresentam melhor 
rigidez/peso que as placas homogêneas analisadas. Já aqueles constituídos com espuma de poliuretano pior rigidez/peso. Constatando-se que nem sempre os materiais compósitos apresentam melhores valores de rigidez/peso.

Todavia, apenas a relação rigidez/peso é insuficiente para determinar o melhor material a ser utilizado. Para isso, é necessário analisar cada situação separadamente, avaliando os demais critérios: custo, espessura máxima, tipos de materiais a serem usados, resistência à fadiga e outras propriedades já citadas neste trabalho.

\section{CONSIDERAÇÕES FINAIS}

Constatou-se que para todos os casos analisados, o erro percentual entre os métodos foi elevado, sendo alguns acima de 50\%. Este fato pode ser atribuído à simplicidade da formulação apresentada em [9]. Percebe-se que em nenhum momento a Equação (4) leva em consideração os coeficientes de Poisson dos materiais constituintes, ou seja, ela assume que as deformações derivadas do efeito de Poisson são constantes ao longo de todo o material. Entretanto as análises realizadas foram em três dimensões e admitiu-se um acoplamento das subregiões nas três direções, ou seja, uma colagem perfeita entre as placas. Com isso, quando se tem materiais com diferentes coeficientes de Poisson ligados e esses materiais estão submetidos a um carregamento os mesmos se deformam de diferentes formas, como se pode observar na Figura 8.

Essa deformação, resultante do efeito Poisson, acarreta o surgimento de uma energia, proveniente das tensões paralelas à direção das placas nas regiões de ligação. Devido a essas tensões, a rigidez efetiva determinada pelo MEC é maior quando comparada à regra da mistura.

No exemplo 2 é estudada a relação rigidez/peso de estruturas sanduíche e comparada com estruturas homogênea. Neste exemplo, verificou-se que em todos os casos a espessura do material homogêneo foi menor que a espessura do material compósito; os materiais com espuma metálica (alumínio light) apresentam melhor rigidez/peso que as placas homogêneas. Já aqueles constituídos com espuma de poliuretano apresentam pior rigidez/peso. Todavia, apenas a relação rigidez/peso é insuficiente para determinar o melhor material a ser utilizado. Para isso, é necessário analisar cada situação separadamente, avaliando os demais critérios: custo, espessura máxima, características de materiais a serem usados, finalidade do projeto, entre outras propriedades.

\section{Agradecimentos}

Os autores agradecem ao Conselho Nacional de Desenvolvimento Científico e Tecnológico (CNPq) pela bolsa concedida dentro do Programa Institucional de Bolsas de Iniciação Científica (PIBIC) - CNPq/UFAL/FAPEAL, e ao Programa de Pós-Graduação em Engenharia Civil da UFAL. 


\section{REFERÊNCIAS}

[1] Almeida, F. P. A. Aplicação do Acoplamento entre o MEC e o MEF para Estudo da Interação Elastoplástica entre o Solo e Estruturas. Tese (Doutorado) - Escola de Engenharia de São Carlos. São Carlos. 2003.

[2] Bacarji, E. Aplicação do Método dos Elementos de Contorno à Análise de Pavimentos de Edifícios. Tese (Doutorado) - Escola de Engenharia de São Carlos, Universidade de São Paulo. São Carlos. 2001.

[3] Becker, A. A. The Boundary Element Method in Engineering. London: McGRAW-HILL Book Company, England, 1992.

[4] Belbute, P. M. G. Estudo do Comportamento em Flexão de Vigas Compósitas Sandwich. Dissertação (Mestrado) - Universidade Técnica de Lisboa. Lisboa. 2010.

[5] Buffoni, S. S. O. Notas de Aula: Resistencia dos Materiais. Escola de Engenharia Industrial Metalúrgica de Volta Redonda. 2008.

[6] Ferreira, A. J. M. Modelos Numéricos para a Análise de Estruturas Laminadas Compósitas e Sandwich. Tese (Doutorado) - Faculdade de Engenharia da Universidade do Porto. Porto. 1996.

[7] Gagliardo, D. P.; Mascia, N. T. Associação Nacional de Tecnologia do Ambiente Construído. Análise de estruturas sanduíche: parâmetros de projeto, Porto Alegre, Dezembro 2010. p. 247-258.

[8] Nemat-Nasser, S E Hori, M. Micromechanics: Overall properties of heterogeneous materials. Amsterdam: Elsevier Science Publishers, 1999.

[9] Roesler, J.; Harders, H.; Baeker, M. Mechanical Behaviour of Engineering Materials: Metals, Ceramics, Polymers and Composites. New York: Springer, 2006.

[10] Vasiliev, V. V.; Morozov, E. V. Mechanics and Analysis of Composite Materials. Elsevier Science Ltd. UK. 2001. 